Equilibrium Outcomes in Two-Stage Amendment Procedures

Author(s): Jeffrey S. Banks

Source: American Journal of Political Science, Vol. 33, No. 1 (Feb., 1989), pp. 25-43

Published by: Midwest Political Science Association

Stable URL: http://www.jstor.org/stable/2111252

Accessed: 18-03-2016 16:04 UTC

Your use of the JSTOR archive indicates your acceptance of the Terms \& Conditions of Use, available at http://www.jstor.org/page/ info/about/policies/terms.jsp

JSTOR is a not-for-profit service that helps scholars, researchers, and students discover, use, and build upon a wide range of content in a trusted digital archive. We use information technology and tools to increase productivity and facilitate new forms of scholarship. For more information about JSTOR, please contact support@jstor.org. 


\title{
Equilibrium Outcomes in Two-Stage Amendment Procedures*
}

\author{
Jeffrey S. Banks, University of Rochester
}

This paper analyzes the set of sophisticated voting outcomes under the following two-stage amendment procedure: the set of alternatives is partitioned into two subsets; one subset is voted on via an amendment procedure at the first stage, followed by the remaining subset at the second stage. The surviving alternatives are then paired at the final vote. This procedure is related to that used in congressional decision making when both a bill and a substitute bill are in order on the floor and hence are open to amendments. It is shown that it is "easier" for alternatives initially considered at the second stage to be the outcome of the voting game than those considered at the first stage. The set of outcomes is contrasted with that generated by the (one-stage) amendment procedure as well as the concept of the uncovered set.

The impact of voting procedures and agendas on the outcomes of collective decision problems has recently become the subject of substantial research by social scientists (see Ordeshook and Schwartz, 1987, and the citations therein). This interest can be seen as deriving from the larger program of analyzing the influence that institutional structures have in determining the outcomes of voting processes; the greater the influence, the greater the amount of "power" held by those in control of these institutional arrangements relative to those deciding at the voting stage of the process. Much of the work on voting procedures and agendas has focused on the amendment voting procedure, where two alternatives are put to a vote, the winner being paired with a new alternative, the winner of that then paired against a new alternative, and so on (Miller, 1980; Shepsle and Weingast, 1984; Banks, 1985). This procedure is attractive for a number of reasons, not the least of which is that it is consistent with the rules in Congress governing the perfecting of a bill through a series of amendments to the bill (Roberts' Rules).

Ordeshook and Schwartz (1987) rightly point out, however, that the amendment voting procedure is only one type, and not necessarily the most prevalent type, of procedure employed by voting bodies such as Congress. They cite the work of congressional scholars in establishing the fact that any number of voting procedures are used in formulating a collective decision. Ordeshook and Schwartz then proceed to show that, given the ability to choose any type of voting procedure, those in charge of such decisions have virtually complete control over the outcome, in that nearly every alternative is the outcome of some procedure and agenda. However, while these results are helpful in establishing upper bounds on the influence of those who decide on procedures and agendas, Ordeshook and Schwartz do not present any evidence concerning whether the procedure they analyze that generates these bounds (i.e., the successive elimina-

*I would like to thank David Austen-Smith, Barry Weingast, and two anonymous referees for valuable comments and suggestions. 
tion procedure) is a prevalent or feasible method of decision making in an organization such as Congress.

A common area of interest to those who study the effects of voting procedures and agendas is the relevance to the processes by which bills before the U.S. Congress become amended prior to a vote on final passage. If a bill is reported out of a committee under an "open rule," members of Congress have the opportunity to propose changes in the bill and to have these changes put to a vote. Both houses of Congress have well-defined rules governing the process by which a bill can be amended, based on the timing and nature of the changes considered. For instance, Rule 19 in the House of Representatives defines "the basic pattern of consideration" for amending a bill on the floor (Sullivan, 1984, p. 32). It states that "when a motion or proposition is under consideration a motion to amend and a motion to amend that amendment shall be in order, and it shall also be in order to offer a further amendment by way of a substitute, to which one amendment may be offered, but which shall not be voted on until the original matter is perfected" (qtd. in Sullivan, 1984). The "amendment by way of a substitute" can simply be considered a substitute bill. Later decisions have modified this rule to allow for substitute amendments to a bill, as well as giving the substitute bill equal status with the original bill in terms of the degree to which it can be amended. The order in which the bill and its substitute are considered is still the same, however; that is, the original bill is perfected prior to the perfecting of the substitute bill. For example, suppose that on the floor of the House there are the following seven alternatives to the status quo: an unamended bill $(b)$, the bill perfected by an amendment $(b a)$, the bill perfected by an amended amendment $(b a a)$, the bill perfected by a substitute amendment $(b s)$, an unamended substitute bill $(s)$, the substitute bill perfected by an amendment ( $s a)$, and the substitute bill perfected by an amended amendment ( $s a a)$. Then the decision procedure, according to Rule 19 and its precedents, is as follows (Sullivan, 1984): (1) $b a$ versus $b a a$ (whether to amend the amendment); (2) the winner at 1 versus $b s$ (whether to substitute for the perfected amendment); (3) the winner at 2 versus $b$ (whether to amend the bill); (4) sa versus $s a a$ (whether to amend the amendment for the substitute bill); (5) the winner at 4 versus $s$ (whether to amend the substitute); (6) the winner at 5 versus the winner at 3 (whether to replace the perfected bill by the perfected substitute bill). In the Senate the process of amending a bill can be quite similar to that of the House. As an example, Bach (1983) describes the case of S.7, the Veteran's Health Care Amendments of 1979. Prior to a vote there were six alternatives to the status quo pending (using the same shorthand as above): $b, b a, b s, s, s a, s a a$. The procedure would be (1) $b a$ versus $b s$; (2) the winner at 1 vs. $b$; (3) $s a$ versus $s a a$; (4) the winner at 3 versus $s$; and (5) the winner at 4 versus the winner at 2 . The resulting bill would then be put to a vote against the status quo.

In both of the above examples, the following structure is present in the voting process: the set of alternatives (excluding the status quo) is partitioned 
into two subsets, namely, those referring to the original bill (including the bill itself) and those referring to the substitute bill. In the first stage of voting, an amendment procedure is employed to perfect the original bill, followed by a second stage in which the substitute bill is perfected via the amendment procedure. Finally, the perfected bill is paired with the perfected substitute to determine the final content of the bill. Thus, a specification of this type of procedure, which we label the two-stage amendment procedure, requires (1) a partition of the alternatives into two subsets and (2) a pair of agendas, or orderings of the alternatives in each subset for consideration via an amendment procedure. This procedure is analyzed in detail in the following sections of the paper. Section 1 contains the necessary hardware for the subsequent analysis, while section 2 reviews the results to date on the outcomes under an amendment procedure. Section 3 examines the two-stage amendment procedure described above, and section 4 extends this analysis to the case where the agenda on the subset considered second is a function of the outcome from the subset considered first. Finally, section 5 concludes with a summary of the results, a discussion of their relevance to the study of voting procedures, and some comments on further research.

\section{Notation, Definitions, Assumptions}

Let $X$ be a finite set of alternatives, $|X|=m, N$ the set of voters, $|N|=n$ odd, and assume that each voter is endowed with a strict preference order $P_{1} \subseteq X$ $\times X$. Let $\Omega(X)$ be the set of all subsets of $X$. The majority preference relation $P$ $\subseteq X \times X$ is defined by

$$
x P y \text { iff }\left|\left\{i \in N: x P_{1} y\right\}\right|>n / 2 .
$$

The assumptions above imply that the relation $P$ is complete and asymmetric; that is, $\forall x, y \in X$, either $x P y$ or $y P x$. In what follows we restrict attention to the majority preference relation rather than the underlying individual preference relations; by McGarvey (1953) any majority preference relation can be generated by an appropriate specification of individual preferences. Define the function $P: X \rightarrow \Omega(X)$ by

$$
P(x)=\{y \in X: y P x\},
$$

analogously define

$$
P^{-1}(x)=\{y \in X: x P y\}
$$

and set

$$
\bar{P}(x)=P^{-1}(x) \cup\{x\} .
$$

We extend the majority preference relation over $X$ to a relation over $\Omega(X)$ as follows: define $\mathscr{D} \subseteq \Omega(X) \times \Omega(X)$ by

$S \mathscr{D} T$ iff $S, T \in \Omega(X)$ and $\forall y \in T \exists x \in S$ such that $x P y$. 
Since the relation $P$ was complete, the relation $\mathscr{D}$ will be as well, although not necessarily asymmetric. This relation is analogous to the "external stability" property of von Neumann-Morgenstern solutions in that every alternative in the complement of the set is "beaten" by some alternative in the set. For any integer $k>1$, define the relation $\mathscr{D}^{k} \subseteq \Omega(X) \times \Omega(X)$ recursively by

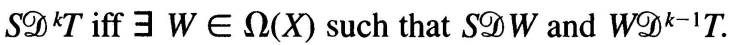

Thus, if $S \mathscr{D}{ }^{k} T$ then $\forall y \in T$ there exists a $k$-step path via the relation $P$ from some $x \in S$ to $y$. Define the subrelation $\mathscr{D}_{m} \subseteq \Omega(X) \times \Omega(X)$, by

$S \mathscr{D}_{m} T$ iff $S \mathscr{D} T$ and $\forall \times \in S \exists y \in T$ s.t. $x P y$ and $y P z, \forall z \in S \backslash\{x\}$.

Alternatively, $S D_{m} T$ if $S \mathscr{T}$ and for no $S^{\prime} \subset S$ is it that $S^{\prime} D T$. Finally, define the relation $C \underline{\subseteq} X X$ by

$$
x C y \text { iff }\{x\} \mathscr{P}(y) \text {. }
$$

This "covering" relation defines a strict partial order on the set $X$. Miller (1980), Shepsle and Weingast (1984), and McKelvey (1986) have examined the set of maximal elements of this relation, the uncovered set $U(X)$, as a solution concept to a number of different game forms. Miller (1980) has shown that this set can be characterized by the following:

Theorem 1 (Miller, 1980): $x \in U(X)$ iff $\{x\} \mathscr{D}^{2} X \backslash\{x\}$.

Thus, alternatives in $U(X)$ can, in two steps, "reach" any other alternative in $X$ via the relation $P$. In subsequent sections we shall see how the solution sets of the various procedures are related to the set $U(X)$.

The class of binary voting procedures we wish to examine can be characterized as occurring on a binary voting tree $\Gamma=(\Lambda, Q ; \psi)$, where $(1)(\Lambda, Q)$ is a topological tree; that is, $\Lambda$ is a set of "nodes" with unique initial node $\lambda_{0} \in \Lambda$, and $Q$ is an asymmetric acyclic binary relation on $\Lambda$ with at most one path between any two nodes, thus describing the "branches" of the tree; (2) $\psi: \Lambda^{t} \rightarrow X$ is an onto function assigning to each terminal node $\lambda \in \Lambda^{t} \subseteq \Lambda$ an alternative from $X$; and (3) each nonterminal node is immediately followed by exactly two nodes. Nonterminal nodes correspond to decision nodes at which a vote is taken between two alternatives, these alternatives being the branches to the next nodes. For all $\lambda \in \Lambda \backslash\left\{\lambda_{0}\right\}$ let $p(\lambda)$ be the alternative in $X$ associated with the branch immediately preceding $\lambda$, and for all nonterminal nodes $\lambda \in \Lambda \backslash \Lambda^{t}$ let $\Gamma(\lambda)$ be the subtree starting from $\lambda$ with assignment $\psi_{\lambda}$ derived from the original assignment $\psi$. All of the procedures analyzed below give rise to voting trees which are uniform, so that the number of decisions required to reach any two terminal nodes is the same, and nonrepetitive, so that this number is precisely $m-1$ (Ordeshook and Schwartz, 1987, provide these and other classifications of binary procedures). For all $\lambda, \lambda^{\prime} \in \Lambda \backslash \Lambda^{t}$ we say that the subtrees beginning with $\lambda$ and $\lambda^{\prime}$ are 
equivalent, $\Gamma(\lambda) \simeq \Gamma\left(\lambda^{\prime}\right)$, if both subtrees are uniform, of the same length, and $\psi_{\lambda}(\cdot)=\psi_{\lambda^{\prime}}(\cdot)$. We assume that individuals adopt sophisticated voting strategies, so that (1) at the final decision nodes (i.e., those followed by only terminal nodes), they vote for the preferred alternative from those associated with the subsequent terminal nodes; (2) at the penultimate decision nodes, they vote for the preferred alternative from those derived from the optimizing behavior of the voters at the final decision nodes; and so on, back up the voting tree. Thus at each decision node we can associate an alternative that will be the ultimate outcome if that decision node is reached; label this outcome $s(\lambda)$. The alternative associated with the initial decision node, $s\left(\lambda_{0}\right)$, will thus be the equilibrium outcome of the game; this outcome we label the sophisticated voting outcome. McKelvey and Niemi (1978) have shown that this outcome is unique for any binary voting procedure based on majority rule.

We can categorize binary voting procedures by the constraints they place on the assignment $\psi(\cdot)$ where, by the assumption of sophisticated voting, this assignment is sufficient to calculate the equilibrium outcome. In the following sections, however, we shall see that techniques exist for calculating the sophisticated voting outcome for the procedures that we examine without recourse to this "voting tree" characterization of the procedures. These techniques will then facilitate the derivation of the set of sophisticated voting outcomes for the procedures. The importance of the voting tree characterizations will be in the identification of segments of the procedures that are similar to that which is well known, namely, the amendment procedure. The next section summarizes the main results of this procedure.

\section{Amendment Procedures}

An amendment voting procedure has the following format: two alternatives are put to a vote, with the winner then facing a new alternative. The winner of this vote is paired with another new alternative, and so forth, until all the alternatives have been considered in a vote at least once. For example, if $X=\{x, y, z$, $a, b, c\}$, then the amendment procedure shown in Figure 1 initially pairs $a$ versus $b$, with the winner paired against $c$, and so on.

Given this scenario, we can characterize an amendment procedure by an agenda $\pi:\{1,2, \ldots, m\} \rightarrow X$, where $\pi$ is a one-one and onto function assigning to each "level" of the procedure an alternative from $X$. Thus, the first vote is between $\pi(1)$ and $\pi(2)$; the winner faces $\pi(3)$; and so forth. In Figure 1 then, $\pi(1)=a, \pi(2)=b$, and so on. Let $\Pi(X)$ be the set of all such orderings of the set $X$. If one were to characterize this procedure by a binary voting tree and adopt the convention that newly recognized alternatives occupy the right branch following a decision node, then the constraint on the assignment $\psi: \Lambda^{t} \rightarrow X$ would be as follows: let $\sigma=2^{m-1}$ be the number of terminal nodes, so that $\Lambda^{t}=\left\{\lambda_{1}, \lambda_{2}, \ldots\right.$, $\left.\lambda_{\sigma}\right\}$; then, 


$$
\begin{aligned}
& \psi\left(\lambda_{2}\right)=\psi\left(\lambda_{4}\right)=\psi\left(\lambda_{6}\right)=\psi\left(\lambda_{8}\right)=\cdots=\psi\left(\lambda_{\sigma}\right) \\
& \psi\left(\lambda_{3}\right)=\psi\left(\lambda_{7}\right)=\psi\left(\lambda_{11}\right)=\cdots=\psi\left(\lambda_{\sigma-1}\right) \\
& \psi\left(\lambda_{5}\right)=\psi\left(\lambda_{13}\right)=\cdots \\
& \psi\left(\lambda_{9}\right)=\psi\left(\lambda_{25}\right)=\cdots
\end{aligned}
$$

and so forth.

Thus in Figure 1 we have that $\psi\left(\lambda_{1}\right)=a, \psi\left(\lambda_{2}\right)=z, \psi\left(\lambda_{3}\right)=y, \psi\left(\lambda_{5}\right)=x$, $\psi\left(\lambda_{9}\right)=c$, and $\psi\left(\lambda_{17}\right)=b$. In general, the relationship between the ordering $\pi$ and the assignment $\psi$ is:

$$
\begin{aligned}
& \psi\left(\lambda_{1}\right)=\pi(1) \\
& \psi\left(\lambda_{2}\right)=\pi(m) \\
& \psi\left(\lambda_{3}\right)=\pi(m-1) \\
& \psi\left(\lambda_{5}\right)=\pi(m-2) \\
& \dot{\cdot} \\
& \dot{\psi} \\
& \psi\left(\lambda_{(\sigma / 2)+1}\right)=\pi(2) .
\end{aligned}
$$

\section{FIGURE 1}

An Amendment Procedure and Majority Preference Relation
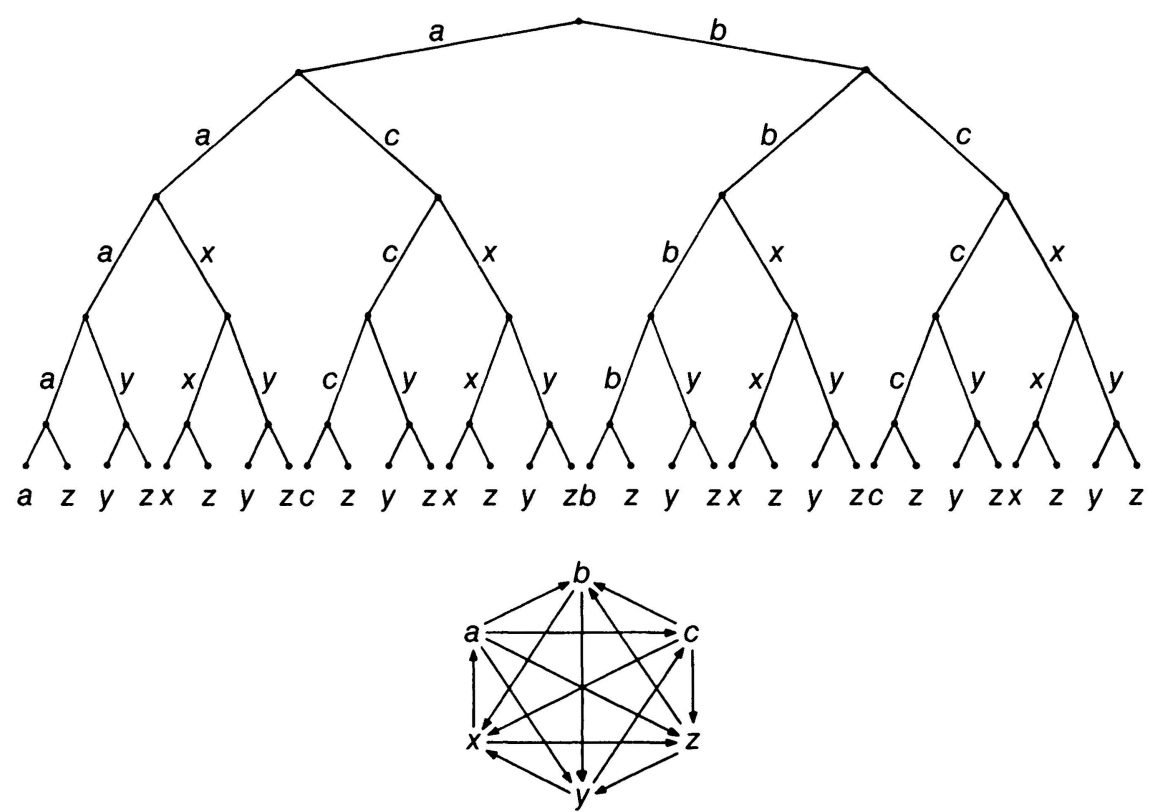
Shepsle and Weingast (1984) have developed an algorithm for calculating the sophisticated voting outcome for an amendment procedure by simply using the "order" characterization of the voting game. This is done by first creating the sophisticated agenda $\mathrm{z}(\pi)=\left\{z_{1}(\pi), z_{2}(\pi), \ldots, z_{m}(\pi)\right\}$, where

$$
\begin{aligned}
& z_{m}(\pi)=\pi(m), \text { and } \forall 1 \leqslant \mathrm{i}<\mathrm{m}, \\
& z_{i}(\pi)=\left[\begin{array}{l}
\pi(i) \text { if } \pi(i) \in P\left(z_{j}\right), \forall j>i, \\
z_{\imath+1} \text { else. }
\end{array}\right]
\end{aligned}
$$

Let $v(X, \pi)$ be the sophisticated voting outcome for the amendment procedure characterized by the ordering $\pi$ over $X$.

Theorem 2 (Shepsle and Weingast, 1984): For all $\pi \in \Pi(X), v(X, \pi)=$ $z_{1}(\pi)$.

Suppose in the example in Figure 1 that the majority preference relation corresponds to that shown below the voting tree, where $y \rightarrow x$ means that $y P x$. Then the sophisticated agenda is $z(\pi)=(c, c, c, x, z, z)$, so that $c$ is the sophisticated voting outcome in Figure 1. Hence, there is a convenient method for calculating the sophisticated voting outcome in an amendment procedure for any ordering of the alternatives.

Let $S^{1}(X)=\underset{\pi \in \Pi(X)}{\bigcup}\{v(X, \pi)\}$. The set $S^{1}(X)$ consists of those alternatives in $X$ which are the sophisticated voting outcome in an amendment procedure for some ordering $\pi$ of $X$. This set can be thought of as the "possibility set" when the decision on the ordering of the alternatives is being made, conditional on $X$ and an amendment procedure being previously chosen. Banks (1985) characterizes this set as follows: for all $x \in X$, let $H(x)=\left\{X^{\prime} \in \Omega(X): P\right.$ is transitive on $X^{\prime}$ and $x$ is $P$-maximal in $\left.X^{\prime}\right\}$.

Theorem 3 (Banks, 1985): $S^{1}(X)=\{x \in X: \exists H \in H(x)$ such that $H \mathscr{D} \backslash \backslash H\}$.

In the above example, $a \in S^{1}(X)$ by the set $\{a, b\}, b \in S^{1}(X)$ by $\{b, x, y\}$, and so forth. The only alternative not in $S^{1}(X)$ is $z$, since the largest set in $H(z)$ is $\{z, b$, $y\}=\bar{P}(z)$, but $\{a\} \mathscr{D}\{z, b, y\}$, so that $a C z$. In general, we have

Theorem 4 (Miller, 1980): $S^{1}(X) \subseteq U(X)$.

Thus, a necessary condition for an alternative to be in $S^{1}(X)$ is that it not be covered by any other alternative in $X$.

Although the above analysis was derived only for amendment procedures, where an alternative can occupy any level in the agenda, it is a simple exercise to extend the analysis to the case where one alternative, $x_{0} \in X$, the status quo, is always voted on last, so that $\pi(m)=x_{0}$. The relevant set of alternatives then becomes the set $P\left(x_{0}\right)$, since by the algorithm of Shepsle and Weingast any alter- 
native that does not beat $\pi(m)$ is, in their terms, "innocuous," or irrelevant to the computation of the sophisticated voting outcome. Thus, the set of sophisticated voting outcomes in this case becomes $S^{1}\left(P\left(x_{0}\right)\right)$ if $P\left(x_{0}\right)$ is nonempty, and it is equal to $\left\{x_{0}\right\}$ if $P\left(x_{0}\right)$ is empty. Alternatively, for all $x \in X$ define the set $H\left(\bullet ; x_{0}\right)$ as

$$
\begin{aligned}
& H\left(x ; x_{0}\right)=\left\{X^{\prime} \in H(x): \min _{P} X^{\prime}=x_{0}\right\}, \text { and then } \\
& S^{1}\left(X ; x_{0}\right)=\left\{x \in X: \exists H \stackrel{\in}{\in} H\left(x ; x_{0}\right) \text { such that } H D X \backslash H\right\} .
\end{aligned}
$$

The following shows that these methods are equivalent.

THEOREM 5: $S^{1}\left(P\left(x_{0}\right)\right)=S^{1}\left(X ; x_{0}\right)$.

Proof: (1) to see: $S^{1}\left(P\left(x_{0}\right)\right) \subseteq S^{1}\left(X ; x_{0}\right)$. Let $X^{\prime}$ be such that Theorem 3 holds for $x$ in $P\left(x_{0}\right)$; then $X^{\prime} \cup\left\{x_{0}\right\} \in H\left(x ; x_{0}\right)$, since $X^{\prime} \subseteq P\left(x_{0}\right)$; and $X^{\prime} \cup$ $\left\{x_{0}\right\} \mathscr{D} X \backslash\left[X^{\prime} \cup\left\{x_{0}\right\}\right]$, since $X^{\prime} \mathscr{D} P\left(x_{0}\right) \backslash X^{\prime}$ and $\left\{x_{0}\right\} \mathscr{D} X \backslash \bar{P}\left(x_{0}\right) \backslash\left\{x_{0}\right\}$. (2) To see: $S^{1}\left(X ; x_{0}\right) \subseteq S^{1}\left(P\left(x_{0}\right)\right)$. Let $X^{\prime} \cup\left\{x_{0}\right\}$ be such that Theorem 3 holds for $x$ in $X$; since $\overline{X^{\prime}} \mathscr{D} P\left(x_{0}\right) \backslash X^{\prime}$ and the relation $P$ is still linear on $X^{\prime}$ the result follows. QED.

For instance, in the above example we have that $S^{1}(X ; b)=\{a, c\}$, while $S^{1}(X ; c)$ $=\{a\}$. In subsequent sections we shall ignore the possible presence of status quo alternatives, since the above result implies that the inclusion of such alternatives poses no analytical problems.

\section{Two-Stage Amendment Procedures}

In this procedure it is assumed that the set $X$ is partitioned into $X_{1}$ and $X_{2}$, where $\left|X_{j}\right|=m_{j}$, and the voting proceeds as follows: two alternatives in $X_{1}$ are paired; the one receiving the majority of votes is then paired against a new alternative from $X_{1}$, and so forth. After the $m_{1}-1$ votes are taken, one alternative from $X_{1}$ remains, and consideration switches to the set $X_{2}$, where again voting proceeds via an amendment procedure. After the $m_{2}-1$ votes are taken on $X_{2}$, the remaining alternatives from $X_{1}$ and $X_{2}$ are then paired, with the prevailing alternative being declared the outcome. Thus we can characterize this procedure by $\left(X_{2} ; \pi_{1}, \pi_{2}\right)$, where $X_{2} \in \Omega(X), X_{1} \equiv X \backslash X_{2}$, and $\left(\pi_{1}, \pi_{2}\right) \in \Pi\left(X_{1}\right) \times \Pi\left(X_{2}\right)$ order the sets $X_{1}$ and $X_{2}$. In Figure $2, X_{1}=\{a, b, c\}, X_{2}=\{x, y, z\}, \pi_{1}(1)=a$, $\pi_{1}(2)=b$, and so on. Let $w\left(X_{2} ; \pi_{1}, \pi_{2}\right)$ denote the sophisticated voting outcome associated with the two-stage procedure $\left(X_{2} ; \pi_{1}, \pi_{2}\right)$.

In the "voting tree" characterization of this procedure, let $\sigma(1)=2^{m_{1}-1}$ be the number of decision nodes immediately following consideration of the alternatives in $X_{1}$, and let $\lambda_{1}, \lambda_{2}, \ldots, \lambda_{\sigma(1)}$ denote these decision nodes. Each of the subtrees $\Gamma\left(\lambda_{\mathrm{i}}\right)$ beginning at these nodes will itself be an amendment procedure with agenda of the form $\pi_{2}^{\prime}\left(\lambda_{i}\right)=\left(\pi_{2}(1), \pi_{2}(2), \ldots, \pi_{2}\left(m_{2}\right), p\left(\lambda_{i}\right)\right)$, where $p\left(\lambda_{i}\right)$ is the remaining alternative from $X_{1}$ associated with the subtree. For example, in the two-stage procedure described in Figure 2, $\pi_{2}^{\prime}\left(\lambda_{1}\right)=(x, y, z, a)$, 


\section{FIGURE 2}

A Two-Stage Amendment Procedure

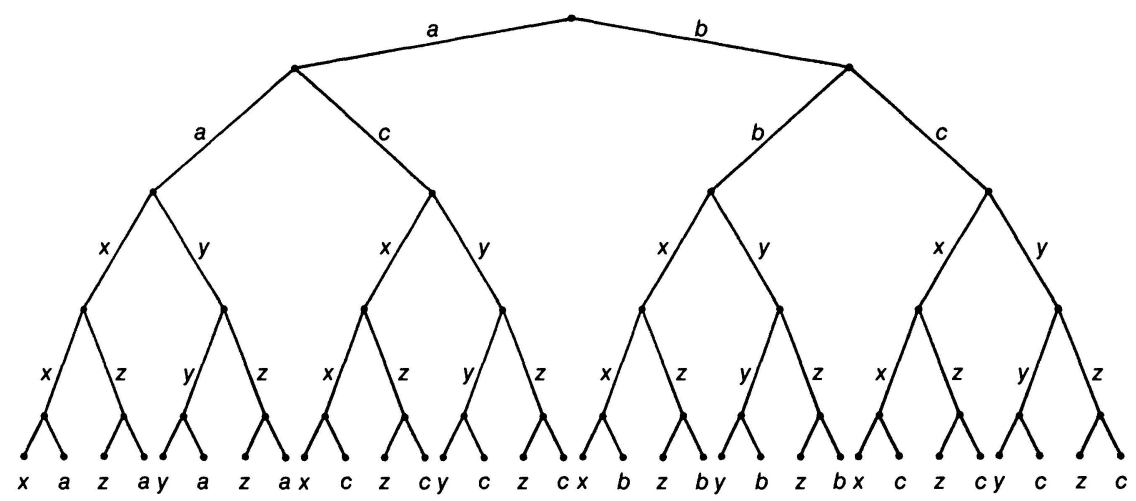

$\pi_{2}\left(\lambda_{2}\right)=(x, y, z, c)$, and so on. Thus, $s\left(\lambda_{i}\right)$, the sophisticated equivalent outcome associated with the node $\lambda_{i}$, will be equal to $v\left(X_{2} \cup\left\{p\left(\lambda_{i}\right)\right\}, \pi_{2}^{\prime}\left(\lambda_{i}\right)\right)$, where recall $v(\cdot, \cdot)$ gives the sophisticated voting outcome for an amendment procedure. Let $\Gamma_{s}=\left(\Lambda_{s}, Q_{s} ; \psi_{s}\right)$ be the binary voting tree derived by replacing the subtrees $\Gamma\left(\lambda_{i}\right)$ defined above with their sophisticated equivalent outcomes. Since $X_{1}$ was itself voted on via an amendment procedure, and the ordering $\pi_{2}$ is not a function of the history of the votes on $X_{1}$, in general it must be the case that

$$
\begin{aligned}
& \Gamma\left(\lambda_{2}\right) \simeq \Gamma\left(\lambda_{4}\right) \simeq \Gamma\left(\lambda_{6}\right) \simeq \cdots \simeq \Gamma\left(\lambda_{\sigma(1)}\right) \\
& \Gamma\left(\lambda_{3}\right) \simeq \Gamma\left(\lambda_{7}\right) \simeq \Gamma\left(\lambda_{11}\right) \simeq \cdots \\
& \Gamma\left(\lambda_{5}\right) \simeq \Gamma\left(\lambda_{13}\right) \simeq \cdots \\
& \Gamma\left(\lambda_{9}\right) \simeq \Gamma\left(\lambda_{25}\right) \simeq \cdots
\end{aligned}
$$

and so forth.

Since equivalent subtrees give rise to the same sophisticated voting outcomes, replacing the nodes $\lambda_{1}, \ldots, \lambda_{\sigma(1)}$ with their sophisticated equivalent alternatives implies that

$$
\begin{aligned}
& \psi_{s}\left(\lambda_{2}\right)=\psi_{s}\left(\lambda_{4}\right)=\cdots \\
& \psi_{s}\left(\lambda_{3}\right)=\psi_{s}\left(\lambda_{7}\right)=\cdots \\
& \psi_{s}\left(\lambda_{5}\right)=\psi_{s}\left(\lambda_{13}\right)=\cdots
\end{aligned}
$$

and so forth.

That is, the voting game $\Gamma_{s}$ will itself have the form of an amendment procedure with associated ordering $\pi_{s}=\left(\pi_{s}(1), \pi_{s}(2), \ldots, \pi_{s}\left(m_{1}\right)\right)$ defined by

$$
\begin{aligned}
& \pi_{s}(1)=\psi_{s}\left(\lambda_{1}\right) \\
& \pi_{s}\left(m_{1}\right)=\psi_{s}\left(\lambda_{2}\right)
\end{aligned}
$$




$$
\begin{aligned}
& \pi_{s}\left(m_{1}-1\right)=\psi_{s}\left(\lambda_{3}\right) \\
& \pi_{s}\left(m_{1}-2\right)=\psi_{s}\left(\lambda_{5}\right) \\
& \cdot \dot{\cdot} \\
& \cdot \\
& \pi_{s}(2)=\psi_{s}\left(\lambda_{(\sigma(1) / 2)+1}\right) .
\end{aligned}
$$

For the example in Figure 2, then, the majority preference relation described in Figure 1 implies that $\psi_{s}\left(\lambda_{1}\right)=x, \psi_{s}\left(\lambda_{2}\right)=\psi_{s}\left(\lambda_{4}\right)=y$, and $\psi_{s}\left(\lambda_{3}\right)=z$, so that the resulting game $\Gamma_{s}$ is as described in Figure 3.

In general there is of course the possibility of redundancies in the order $\pi_{s}$; that is, $\pi_{s}(i)=\pi_{s}(j)$, some $i \neq j$. However, by the algorithm of Shepsle and Weingast in the preceding section, this can be remedied by simply removing $\pi_{s}(i)$ if $i<j$ or $\pi_{s}(j)$ if $j<i$, creating an agenda of length less than $m_{1}$. Thus, given a partition $X_{1}$ and $X_{2}$ of $X$ and orderings $\pi_{1}$ and $\pi_{2}$, one can calculate the sophisticated voting outcome $w\left(X_{2} ; \pi_{1}, \pi_{2}\right)$ by initially creating a first-order sophisticated agenda $y\left(X_{2} ; \pi_{1}, \pi_{2}\right)=\left\{y_{1}(\cdot), y_{2}(\cdot), \ldots, y_{m_{1}}(\cdot)\right)$, defined by $y_{i}\left(X_{2} ; \pi_{1}\right.$, $\left.\pi_{2}\right)=v\left(X_{2} \cup\left\{\pi_{1}(i)\right\}, \pi_{2}^{\prime}\left(\pi_{1}(i)\right)\right)$, where $\pi_{2}^{\prime}(\cdot)$ is defined as above. Applying the algorithm of Shepsle and Weingast to the agenda $y(\cdot)$ to generate the agenda $z(y(\cdot))$, we get,

TheOREM 6: For all $X_{2} \in \Omega(X)$ and $\left(\pi_{1}, \pi_{2}\right) \in \Pi\left(X_{1}\right) \times \Pi\left(X_{2}\right)$, $w\left(X_{2}, \pi_{1}, \pi_{2}\right)=z_{1}\left(y\left(X_{2} ; \pi_{1}, \pi_{2}\right)\right)$.

In Figure 2, $y(\cdot)=(x, z, y)$, so that the sophisticated voting outcome of this twostage procedure is $z$. Thus, for two-stage amendment procedures there exists an algorithm for calculating the sophisticated voting outcome analogous to the process developed by Shepsle and Weingast for solving the (one-stage) amendment procedures. One-stage amendment procedures necessitate the use of the Shepsle and Weingast algorithm on one agenda of length $m$, while two-stage procedures require solving $m_{1}$ agendas of length $m_{2}+1$ and one agenda of length (at most) $m_{1}$.

As in the preceding section, we are interested in characterizing the set of alternatives that constitutes the "possibility set" when a two-stage amendment procedure is employed to reach a collective decision. Let $S^{2}(X)$ be the set of alternatives which are sophisticated voting outcomes under some partition of $X$ and some ordering of the elements of the partition. By Theorem 6 it is clear that alternatives in $S^{2}(X)$ have the property that they are also in $S^{1}\left(X^{\prime}\right)$ for some specific set $X^{\prime} \subseteq \mathrm{X}$. The set $X^{\prime}$ is the set of sophisticated voting outcomes derived from the subtrees defined by $X_{2}$ and $\pi_{2}$, while the order in which they are placed in the agenda $y(\cdot)$ is defined by $\pi_{1}$. Given $X_{2}, \pi_{2}$, define

$$
R\left(X_{2} ; \pi_{2}\right)=\bigcup_{x \in X_{1}}\left\{v\left(X_{2} \cup\{x\}, \pi_{2}^{\prime}(x)\right)\right\} \text {. }
$$

THEOREM 7: $x \in S^{2}(X)$ iff $\exists X_{2} \in \Omega(X), \pi_{2} \in \Pi\left(X_{2}\right)$ such that $x \in S^{1}\left(R\left(X_{2} ; \pi_{2}\right)\right)$. 
FIGURE 3

The Reduced Voting Game Derived from Figure 2

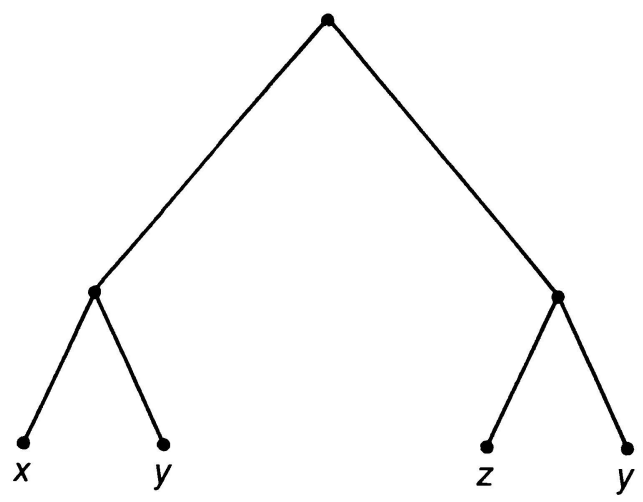

Proof: (1) (nec.) By Theorem 6,

$$
R\left(X_{2} ; \pi_{2}\right)=\bigcup_{i=1}^{\mathrm{m}_{1}}\left\{y_{i}\left(X_{2} ; \pi_{1}, \pi_{2}\right)\right\} ;
$$

thus the result follows from Theorem 4. (2) (suff.) Let $\left|R\left(X_{2} ; \pi_{2}\right)\right|=m_{\mathrm{r}}$, and let $\pi_{x} \in \Pi\left(R\left(X_{2} ; \pi_{2}\right)\right)$ be an agenda on $R\left(X_{2} ; \pi_{2}\right)$ such that $x$ is the sophisticated voting outcome. We construct an order on the set $X_{1}$ as follows: $\forall b \in R\left(X_{2} ; \pi_{2}\right)$ $\cap X_{2}$, define $A(B)=\left\{a \in X_{1}: v\left(X_{2} \cup\{a\}, \pi_{2}^{\prime}(a)\right)=b\right\}$, and let $a(b)$ be a selection from $A(b)$. For $0 \leqslant \mathrm{j} \leqslant \mathrm{m}_{\mathrm{r}}-1$, define

$$
\pi_{1}\left(m_{1}-j\right)=\left\{\begin{array}{lll}
\pi_{x}\left(m_{r}-j\right) & \text { if } & \pi_{x}\left(m_{r}-j\right) \in X_{1} \\
a\left(\pi_{x}\left(m_{r}-j\right)\right) & \text { else. }
\end{array}\right\}
$$

Order the remaining alternatives from $X_{1}$ in any fashion from 1 to $m_{1}-m_{r}$. Then, in the first-order equivalent agenda $\left(y_{m_{1}-m_{r}+1}, \ldots, y_{m_{1}}\right)=\left(\pi_{x}(1), \pi_{x}(2)\right.$, $\left.\ldots, \pi_{x}\left(m_{r}\right)\right)$, and by definition of the set $R(\cdot)$ all remaining alternatives in $y$ are equal to some alternative in $\pi_{x}$. Thus, by Theorem 6 the sophisticated voting outcome will be $x$. QED.

Corollary 1: $\{x\}=S^{2}(X)$ iff $P(x)=\phi$.

Proof: (1) (nec.) Suppose not; let $y P x$ and put $x \in X_{1}, y \in X_{2}$. Then $x \notin$ $R\left(X_{2} ; \pi_{2}\right)$ for any $\pi_{2}$. Contradiction. (2) (suff.) $\forall X_{2}, \pi_{2}, x \in R\left(X_{2} ; \pi_{2}\right)$; further, $x P y, \forall y \in R\left(X_{2} ; \pi_{2}\right), y \neq x$. Thus $\{x\}=S^{1}\left(R\left(X_{2} ; \pi_{2}\right)\right)$. QED.

The characterization in Theorem 7 does not by itself provide much in the way of an intuitive "feel" for the set $S^{2}(X)$. However, the following results show that Theorem 7 can be used to generate necessary and sufficient conditions for an alternative to be in $S^{2}(X)$, where these conditions themselves provide interesting contrasts to other solution concepts. 
Theorem 8: $x \in S^{2}(X)$ if (1) $x \in U(X)$, or (2) $x \in S^{1}\left(X^{\prime}\right)$, where $X^{\prime} \mathscr{D}_{m}$ $X \backslash X^{\prime}$.

Proof: (1) Set $X_{2}=P^{-1}(x)$ and let $\pi_{2}$ be an arbitrary ordering of $X_{2}$. Then clearly $x=v\left(X_{2} \cup\{x\}, \pi_{2}^{\prime}(x)\right)$, so that $x \in R\left(X_{2} ; \pi_{2}\right)$. Hence, by Theorems 4 and 7, $x \in U(X)$ implies $x \in S^{1}\left(R\left(X_{2} ; \pi_{2}\right)\right)$, which implies $x \in S^{2}(X)$. (2) Set $X_{2}=X^{\prime}$; since $X^{\prime} \mathscr{D} \backslash X^{\prime}, R\left(X_{2} ; \pi_{2}\right) \subseteq X^{\prime}$, for any $\pi_{2}$. But since each $y \in X^{\prime}$ defeats at least one alternative in $X_{1}=X \backslash X^{\prime}$ that no other defeats, it must be that $X^{\prime} \subseteq R\left(X_{2} ; \pi_{2}\right)$ as well. Therefore, $R\left(X_{2} ; \pi_{2}\right)=X^{\prime}$, for any $\pi_{2}$, so that since $x \in S^{1}\left(X^{\prime}\right)$, the result follows. QED.

Corollary 2: $S^{1}(X) \subseteq S^{2}(X)$.

PROOF: Follows from Theorems 4 and 8. QED.

Thus, the "possibility set" under a two-stage amendment procedure is larger (by inclusion) than that under a one-stage amendment procedure. Further, as with the set $S^{1}(X)$, if $P(x) \neq \phi \forall x \in X$, then $S^{2}(X)$ contains at least three alternatives.

An interesting feature of the two-stage amendment procedure is that one can analyze the "possiblity set" at the intermediate stage where the partition of $X$ has been chosen but the ordering of the alternatives in each set has not. This also allows for more insight into the asymmetric positions of alternatives in the set $X_{1}$ and those in $X_{2}$ and for the derivation of necessary conditions for an alternative to be in the set $S^{2}(X)$. Let $T\left(X_{2}\right)$ be the set of sophisticated voting outcomes for any orderings of the sets $X_{2}$ and $X_{1} \equiv X \backslash X_{2}$. From the above definitions, then,

$$
T\left(X_{2}\right)=\underset{\pi_{1}, \pi_{2}}{\cup}\left\{w\left(X_{2} ; \pi_{1}, \pi_{2}\right)\right\} .
$$

THEOREM 9: For all $X_{2} \in \Omega(X), x \in X_{1} \cap T\left(X_{2}\right)$ only if (1) $\{x\} \mathscr{D} X_{2}$ and (2) $x \in U(X)$.

Proof: (1) If $\exists y \in X_{2}$ such that $y P x$, then $x \notin R\left(X_{2} ; \pi_{2}\right), \forall \pi_{2} \in \Pi\left(X_{2}\right)$. (2) Suppose not; let $y C x$. By (1), then $y \in X_{1}$. Since $y \mathscr{D} \bar{P}(x), x \in R\left(X_{2}\right.$; $\left.\pi_{2}\right)$ implies $y \in R\left(X_{2} ; \pi_{2}\right)$. But then $y$ covers $x$ in the set $R\left(X_{2} ; \pi_{2}\right)$ as well, so that by Theorem 4, $x \notin S^{1}\left(R\left(X_{2} ; \pi_{2}\right)\right)$. Contradiction. QED.

Thus, if an alternative is in the first set to be considered, it is necessary for that alternative to be both uncovered in $X$ and majority preferred to every alternative in the second set if it is to be the equilibrium outcome. The logic of the latter condition is that the set $X_{1}$ consists of the set of "status quos" for the amendment agendas $\pi_{2}^{\prime}(\cdot)$ so that, as was shown at the end of the previous section, these will be the sophisticated voting outcomes of their respective agendas only if they defeat all the other alternatives in the agenda.

Theorem 10: For all $X_{2} \in \Omega(X), x \in X_{2} \cap T\left(X_{2}\right)$ only if (1) $X_{2} \mathscr{D} X_{1}$, (2) $y C x$ for no $y \in X_{2}$, and (3) $x P z$, some $z \in X_{1}$. 
Proof: (1) Suppose not; then $\exists y \in X_{1}$ such that $y \mathscr{D} X_{2}$, and let $Y$ denote the set of alternatives in $X_{1}$ for which this is true. Then $Y \subseteq R\left(X_{2} ; \pi_{2}\right)$, and $\forall z \in R\left(X_{2} ; \pi_{2}\right) \backslash Y$ and $y \in Y, y P z$. Thus $z \notin U\left(R\left(X_{2} ; \pi_{2}\right)\right)$, so that by Theorem 4, $S^{1}\left(R\left(X_{2} ; \pi_{2}\right)\right) \subseteq Y \subseteq X_{1}$. Contradiction. (2) If $y C x$, then by Theorems 4 and 6, $x \notin R\left(X_{2} ; \pi_{2}\right), \forall \pi_{2} \in \Pi\left(X_{2}\right)$. (3) If not then $x \notin R\left(X_{2}\right.$; $\left.\pi_{2}\right), \forall \pi_{2} \in \Pi\left(X_{2}\right)$. QED.

Alternatives in $X_{2}$ have a somewhat "easier" necessary condition to meet in that they only have to defeat some alternative in $X_{1}$ and not be covered by any other alternative in $X_{2}$.

Corollary 3: $x \in S^{2}(X)$ only if $\{x\} D^{3} X \backslash\{x\}$.

Proof: If $x=w\left(X_{2} ; \pi_{1}, \pi_{2}\right)$ and $x \in X_{1}$, then by Theorems 1 and 9.2, $\{x\} \mathscr{D}^{2} X \backslash\{x\}$. If $x \in X_{2}$, then by Theorem 10.2, $\{x\} \mathscr{D}^{2} X_{2} \backslash\{x\}$, and by Theorem 10.1, $X_{2} \mathscr{D} X_{1}$. QED.

This result contrasts nicely with Theorems 1 and 4: if $x \in S^{1}(X)$, then $x$ can reach every other alternative in at most two steps, while if $x \in S^{2}(X), x$ can reach every other alternative in at most three steps.

Corollary 4: For all $X_{2} \in \Omega(X)$,

$T\left(X_{2}\right) \subseteq\left\{\begin{array}{l}X_{2} \text { if } X_{2} \mathscr{D} X_{1} \\ X_{1} \text { else. }\end{array}\right\}$

PROOF: Follows from Theorems 9 and 10. QED.

Thus, once the partition of the set $X$ has been fixed, all of the sophisticated voting outcomes will be either in $X_{1}$ or $X_{2}$, regardless of the ordering of the alternatives. Note that so far we have been actually assuming that both a partition and an ordering of the elements of the partition have been chosen. Suppose now that a partition $X^{\prime}, X \backslash X^{\prime}$ has been decided upon, but not whether $X^{\prime}=X_{1}$ or $X^{\prime}=X_{2}$. The following then gives a necessary condition for the set of sophisticated voting outcomes to be independent of the order in which the sets $X^{\prime}$ and $X \backslash X^{\prime}$ are considered.

Corollary 5: $T\left(X^{\prime}\right)=T\left(X \backslash X^{\prime}\right)$ only if (1) $X^{\prime} \mathscr{D} X \backslash X^{\prime}$ and $\sim\left[X \backslash X^{\prime} \mathscr{D} X^{\prime}\right]$ or (2) $X \backslash X^{\prime} \mathscr{D} X^{\prime}$ and $\sim\left[X^{\prime} \mathscr{D} \backslash X^{\prime}\right]$.

PRoOF: Follows from Corollary 4. QED.

Since the relation $\mathscr{D}$ is complete, the first part of (1) or the first part of (2) must hold. If neither condition holds, then all of the sophisticated voting outcomes will be from the set of alternatives considered second.

The above analysis has assumed no constraints on $m_{1}$ and $m_{2}$; that is, the size of the sets $X_{1}$ and $X_{2}$. It is possible however that in examining two-stage amendment agendas one should require that the sets $X_{1}, X_{2}$ and their related 
orderings $\pi_{1}, \pi_{2}$ each nontrivially reduce the set of alternatives under consideration. This then implies the restriction that $m_{\imath}>1, i=1,2$. Indeed, if either $m_{1}$ $=1$ or $m_{2}=1$, it is easy to see that the resulting procedure will be equivalent to an amendment procedure, so that the differentiation between the two procedures is lost. Adding the above restriction changes none of the preceding results except for Corollary 2; that is, it need not be the case that every equilibrium outcome in a one-stage amendment procedure is also an equilibrium outcome in a two-stage procedure. To see this, suppose that $P^{-1}(x)=\{y\}$, and $P^{-1}(y)=X \backslash\{x, y\}$. Then $x \in S^{1}(X)$ by the order $\pi(m)=y, \pi(m-1)=x$, but $x \notin S^{2}(X)$ under the above restriction. If $x \in X_{1}$, then it must be that $\{y\}=X_{2}$, by Theorem 9, which implies $m_{2}=1$. If $x \in X_{2}$, then by Theorem $10 y \in X_{2}$ as well. But then $z P x$, $\forall z \in X_{1}$, so that $x \notin R\left(X_{2} ; \pi_{2}\right)$, for any $\pi_{2} \in \Pi\left(X_{2}\right)$, proving the claim. Thus, if one requires that each element of the partition contain at least two alternatives, the notion that a two-stage amendment procedure allows for more (by inclusion) alternatives to be in the possibility set than a one-stage amendment procedure does not hold. Without this restriction, however, we have,

THEOREM 11: If $|X| \equiv m \leqslant 5$, then $S^{2}(X)=S^{1}(X)$.

Proof: By Corollary 2, we need to show that $S^{2}(X) \subseteq S^{1}(X)$. Let $x \in$ $S^{2}(X)$, but $x \notin S^{1}(X)$. By the discussion above we consider only $m_{1}=2$ or $m_{1}=3$, so suppose $m_{1}=2$. If $x \in X_{1}$ for the procedure where $x$ is the outcome, then by Theorem $10\{x\} \not X_{2}$, and hence $y P x$, where $\{x, y\}=X_{1}$. Then $y \notin R\left(X_{2} ; \pi_{2}\right)$ so $\exists z \in X_{2}$ such that $z P y$. Thus $\{x, z\} \in H(x)$ and $\{x, z\} \nsupseteq X \backslash\{x, z\}$, which contradicts $x \notin S^{1}(X)$. If $x \in X_{2}$, then by Theorem 10 $\exists w \in X_{1}$ such that $x P w$; let $\{y, w\}=X_{1}$. Since $\left|R\left(X_{2} ; \pi_{2}\right)\right|=2, \exists z \in X_{2}$ such that $z P w$ and $x P z$. Then $\{x, y, z\} \in H(x)$, and $z P w$ and either $x P v$ or $y P v$, for $v=X_{2} \backslash\{x, z\}$, so that $x \in S^{1}(X)$. If $m_{1}=3$, then $\{x\} D X_{2}$ if $x \in X_{1}$, so that $\left\{x, X_{2}\right\} \in H(x)$, since $X_{2}$ contains only two alternatives. Thus if $x=$ $w\left(X_{2} ; \pi_{1}, \pi_{2}\right)$, then it must be that $\forall y \in X_{1}$ either $x P y$ or $z P y$, some $z \in X_{2}$, so that $x \in S^{1}(X)$. If $x \in X_{2}$, then $X_{2} \equiv\{x, z\} \mathscr{D} X_{1}$, so that if $x P z$ then $\{x, z\}$ $\in H(x)$ and if $z P x$ then $\exists w \in X_{1}$ such that $x P w$, and $\{x, w\} D X \backslash\{x, w\}$, implying $x \in S^{1}(X)$. QED.

The example in Figure 2 shows that this bound is tight, since $m=6$, but $S^{1}(X)=X \backslash\{z\}$, while $S^{2}(X)=X$.

\section{Two-Stage Conditional Amendment Procedures}

In the above analysis we saw that, since the alternatives in $X_{1}$ were voted on by an amendment procedure, it implied that the subtrees beginning with the first votes in $X_{2}$ were all amendment procedures with the additional feature that the agendas for these amendment procedures were identical except for the last alternative. Suppose now that we allowed the agenda on the set $X_{2}$ be a function of the outcome of the voting on the set $X_{1}$, so that a procedure would be character- 
ized by $X_{2} \in \Omega(X), \pi_{1} \in \Pi\left(X_{1}\right)$, and a function $\pi_{2}: X_{1} \rightarrow \Pi\left(X_{2}\right)$ assigning to every possible outcome in $X_{1}$ an ordering of the set $X_{2}$; let $\Pi_{f}\left(X_{2}\right)$ be the set of such functions and $w\left(X_{2} ; \pi_{1}, \pi_{2}(\cdot)\right)$ be the sophisticated voting outcome for any two-stage conditional amendment procedure. For the subtrees beginning at $X_{2}$, the voting still follows an amendment procedure, but now the orderings of the alternatives in $X_{2}$ may differ. However, since the ordering of the set $X_{2}$ is a function only of the outcome of the vote in $X_{1}$ and not a function of the entire history of votes in $X_{1}$, the equivalences among the subtrees $\Gamma\left(\lambda_{i}\right)$, and hence their sophisticated equivalent outcomes, will still hold. Therefore, the binary voting tree $\Gamma_{s}$ obtained by replacing the subtrees with their sophisticated equivalent outcomes will again be an amendment procedure. Thus, to compute the sophisticated voting outcome for this two-stage conditional amendment procedure $\left(X_{2} ; \pi_{1}, \pi_{2}(\cdot)\right)$, an analogous process to the one used in the above section will apply. Define the first-order sophisticated agenda $y\left(X_{2} ; \pi_{1}, \pi_{2}(\cdot)\right)=\left(y_{1}(\cdot)\right.$, $\left.y_{2}(\cdot), \ldots, y_{m_{1}}(\cdot)\right)$ by

$$
y_{i}\left(X_{2} ; \pi_{1}, \pi_{2}(\cdot)\right)=v\left(X_{2} \cup\left\{\pi_{1}(i)\right\}, \pi_{2}^{\prime}\left(\pi_{1}(i)\right)\right),
$$

where now $\pi_{2}^{\prime}\left(\pi_{1}(i)\right)=\left(\pi_{2}\left(1 ; \pi_{1}(i)\right), \pi_{2}\left(2 ; \pi_{1}(i)\right), \ldots, \pi_{2}\left(m_{2} ; \pi_{1}(i)\right), \pi_{1}(i)\right)$ and $v(\cdot)$ is the sophisticated voting outcome associated with the set and order which are its arguments. Applying the Shepsle and Weingast algorithm $z(y(\cdot))$ to the agenda $y(\cdot)$, then, the equivalence theorem for this procedure becomes

THEOREM 12: For all $X_{2} \in \Omega(X), \pi_{1} \in \Pi\left(X_{1}\right), \pi_{2}(\cdot) \in \Pi_{f}\left(X_{2}\right)$, $w\left(X_{2} ; \pi_{1}, \pi_{2}(\cdot)\right)=z_{1}\left(y\left(X_{2} ; \pi_{1}, \pi_{2}(\cdot)\right)\right.$.

As with two-stage amendment procedures, one needs to solve for $m_{1}$ agendas of length $m_{2}+1$ and one agenda of length at most $m_{1}$ to calculate the sophisticated voting outcome for two-stage conditional amendment procedures.

Allowing the order on the set $X_{2}$ to vary across the outcomes of the $X_{1}$ votes implies that alternatives in the set $S_{c}^{2}(X)$ of alternatives which are sophisticated voting outcomes of some two-stage conditional amendment agenda also are in $S^{1}\left(X^{\prime}\right)$ for some $X^{\prime} \subseteq X$, as before, where now the set $X^{\prime}$ can be derived from different orderings on the set $X_{2}$. For any $X_{2}, \pi_{2}(\cdot)$, define

$$
R\left(X_{2} ; \pi_{2}(\cdot)\right)=\bigcup_{x \in X_{1}}\left\{v\left(X_{2} \cup\{x\} ; \pi_{2}^{\prime}(x)\right)\right\} .
$$

As in the above section, this is the set of alternatives in the first-order sophisticated agenda. In the statement of a characterization theorem analogous to that of Theorem 7, however, the ability to choose a different ordering of $X_{2}$ for different alternatives in $X_{1}$ implies that the set $R(\cdot)$ can include an alternative which is the equilibrium outcome for some $x \in X_{1}$ and some agenda on $X_{2} \cup\{x\}$ where $x=$ $\pi_{2}\left(m_{2}+1\right)$. This then implies that the characterization of the set $S_{c}^{2}(X)$ can be generated without reference to an ordering $\pi_{2}$, in contrast to the characterization of the set $S^{2}(X)$. 
THEOREM 13: $x \in S_{c}^{2}(X)$ iff $\exists X_{2} \in \Omega(X)$ s.t. $x \in S^{1}$

$$
\left(\bigcup_{x \in X_{1}} S^{1}\left(X_{2} \cup\{x\} ; x\right)\right) \text {. }
$$

The proof of Theorem 13 follows an analogous logic to that of Theorem 7 and is omitted. An immediate result is,

Corollary 6: $S^{2}(X) \subseteq S_{c}^{2}(X)$.

Thus, the sufficient conditions given in Theorem 8 will also hold for the set $S_{c}^{2}(X)$. Furthermore, the necessary conditions in Theorems 9 and 10 will be necessary conditions for the set $S_{c}^{2}(X)$ as well, since none of these conditions were functions of orderings of the set $X_{2}$. Hence, we state the following results without proof. Define

$$
T_{c}\left(X_{2}\right)=\underset{\pi_{1}, \pi_{2}(\cdot)}{\cup}\left\{w\left(X_{2} ; \pi_{1}, \pi_{2}(\cdot)\right)\right\}
$$

as the set of equilibrium outcomes for a fixed partition $X_{2}, X_{1} \equiv X \backslash X_{2}$.

Theorem 14: For any $X_{2} \in \Omega(X), x \in X_{1} \cap T_{c}\left(X_{2}\right)$ only if (1) $\{x\} \not X_{2}$ and (2) $x \in U(X)$.

Theorem 15: For any $X_{2} \in \Omega(X), x \in X_{2} \cap T_{c}\left(X_{2}\right)$ only if (1) $X_{2} \mathscr{D} X_{1}$;

(2) $y C x$ for no $y \in X_{2}$; and (3) $x P z$ for some $z \in X_{1}$.

In relation to the set $S^{2}(X)$, we have the following analogous result to that of Theorem 11.

TheOrem 16: If $|X| \leqslant 5$, then $S^{2}(X)=S_{c}^{2}(X)$.

Proof: By Corollary 6 we need to show $S_{c}^{2}(X) \subseteq S^{2}(X)$, so let $x=w\left(X_{2} ; \pi_{1}\right.$, $\left.\pi_{2}(\cdot)\right)$. If $m_{1}=1$ or 4 , then the result follows trivially, so let $m_{1}=3$. If $x \in$ $X_{1}$ then $\{x\} \not X_{2}$, so that $\forall \pi_{2}(\cdot) \exists Y \subseteq X_{1}$ such that $Y \subseteq R\left(X_{2} ; \pi_{2}(\cdot)\right)$ and $y P z, \forall y \in Y$ and $z \in R(\cdot) \backslash Y$, where $x \in Y$. Thus the outcome will not be a

FIGURE 4

A Majority Preference Relation Where $S^{2}(X) \subset S_{c}^{2}(X)$

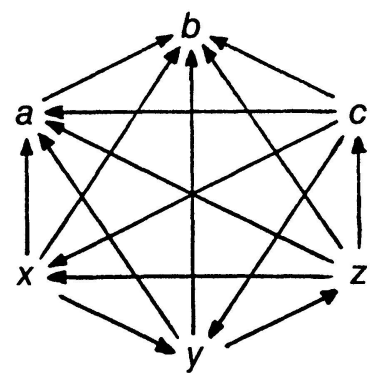


FIGURE 5

A Two-Stage Conditional Amendment Procedure

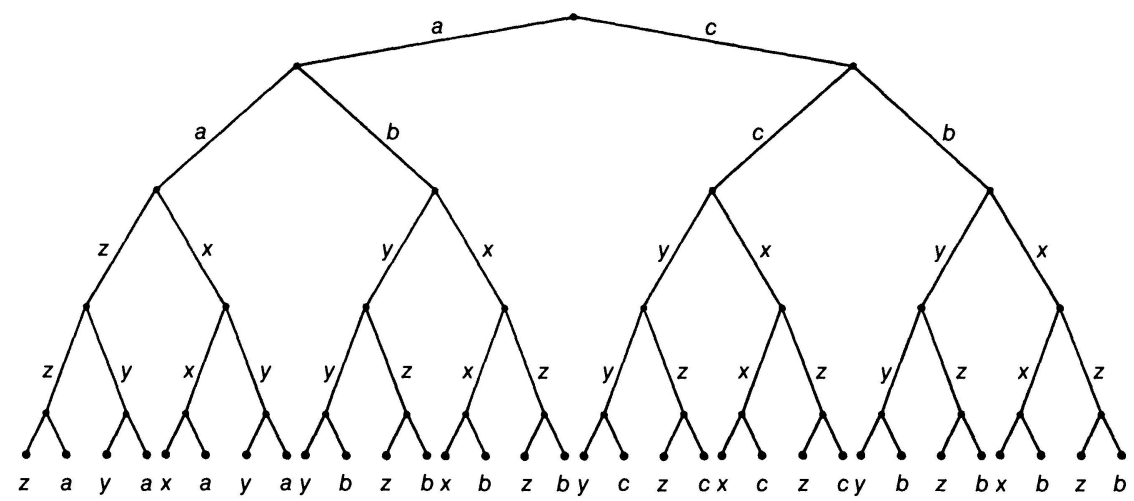

function of $\pi_{2}(\cdot)$. If $x \in X_{2}$, then $X_{2} \equiv\{x, z\} \not X_{1}$, so that setting $\pi_{2}$ equals to the ordering $\pi_{2}(\cdot)$ which implies $x \in R(\cdot)$ will imply $x=w\left(X_{2} ; \pi_{1}, \pi_{2}\right)$. If $m_{1}=2$, then again if $x \in X_{1}$ the result follows, while if $x \in X_{2}$, then $X_{2}$ $\equiv\{x, y, z\} \mathscr{D}\{a, b\} \equiv X_{1}$, and let $x P a, y P b, x P y$, and $\{x, y\}=R\left(X_{2} ; \pi_{2}(\cdot)\right)$. If $x P z$ or $y P z$, then by defining $X_{1}^{\prime}=X_{1} \cup\{z\}$ and setting $\pi_{2}$ equal to the ordering which implies $x \in R(\cdot)$, the result follows for $X_{2}^{\prime}=\{x, y\}$. If ${ }_{z} P x$ and $z P y$, then $\{x, y\}=R(\cdot)$ implies $a P z$ and $b P z$, so that again setting $\pi_{2}$ equal to the above ordering implies $x=w\left(X_{2} ; \pi_{1}, \pi_{2}\right)$, so that $x \in$ $S^{2}(X)$. QED.

To see that the bound in the above theorem is tight, consider the majority preference relation given in Figure 4. We see that $S^{1}(X)=\{c, z, y\}$, and that $c C x$. Suppose that $x \in S^{2}(X)$; by Theorem 9, then $x \in X_{2}$ if $x=w\left(X_{2} ; \pi_{1}, \pi_{2}\right)$, and by Theorem 10, it must be that $c \in X_{1}$ and $z \in X_{2}$. Further, if $z \in X_{2}$ then $y \in X_{2}$ as well, since if not, then $R\left(X_{2} ; \pi_{2}\right)=\{x, z\}$ and $z P x$, so that $x \neq$ $w\left(X_{2} ; \pi_{1}, \pi_{2}\right)$. So suppose $X_{1}=\{a, b, c\}$ and $X_{2}=\{x, y, z\}$, and let $\pi_{2}^{\prime}$ be the ordering which gives $x=v\left(X_{2} \cup\{a\}, \pi_{2}^{\prime}\right)$. Then it must be that $\pi_{2}^{\prime}(3)=y$, since otherwise $x$ would not be the outcome. But then $z=v\left(X_{2} \cup\{i\}, \pi_{2}^{\prime}\right)$ for $i=b, c$, so that $\{x, z\}=R\left(X_{2} ; \pi_{2}\right)$, implying that $x \neq w\left(X_{2} ; \pi_{1}, \pi_{2}\right)$ and $x \notin S^{2}(X)$. Further, any other partition of $X$ will suffer from a similar drawback. However, $x$ $i s$ in $S_{c}^{2}(X)$ by the following: $X_{1}=\{a, b, c\}, X_{2}=\{x, y, z\}, \pi_{2}(a)=(z, x, y)$, $\pi_{2}(b)=(x, y, z)=\pi_{2}(c)$, and $\pi_{1}=(a, c, b)$; see Figure 5. To see that $x=$ $w\left(X_{2} ; \pi_{1}, \pi_{2}(\cdot)\right)$, note that now $x=v\left(X_{2} \cup\{a\}, \pi_{2}(a)\right), y=v\left(X_{2} \cup\{b\}, \pi_{2}(b)\right)$, and $z=v\left(X_{2} \cup\{c\}, \pi_{2}(c)\right)$, and that by Theorem 12 the first-order sophisticated agenda is $(x, z, y)$. Thus, since $y P z$ and $x P y, x$ is the equilibrium outcome for this two-stage conditional amendment procedure. 


\section{Conclusion}

This paper has analyzed the two-stage amendment procedure as a method for solving collective choice problems in voting bodies such as Congress. The main results of the paper are as follows:

1. There exists a well-defined algorithm for computing the sophisticated voting outcome for any two-stage amendment procedure. This algorithm is based on that developed by Shepsle and Weingast (1984) for the one-stage amendment procedure.

2. The set of possible outcomes under a two-stage amendment procedure is larger (by inclusion) than that under a one-stage amendment procedure. That is, in congressional parlance, an alternative that might not have been an outcome when all such alternatives are in the form of amendments to an original bill (or the bill itself) might now be the outcome when the alternatives are divided into two groups, namely those referring to the original bill and those referring to a substitute bill.

3. It is "easier" for alternatives considered in the second stage to become the final outcome than it is for alternatives in the first stage. This result is related to congressional decision making by the rule specifying the perfection of the original bill prior to the perfection of any substitute bill. Thus, in the language of the above analysis, the original bill and its amendments are $X_{1}$ and the substitute bill and its amendments are $X_{2}$. The analysis above has shown that the conditions for an alternative considered as a substitute bill or one of its amendments to be the final outcome are weaker than those for the original bill or one of its amendments. This would seem to give a strategic advantage to those who propose and amend the substitute bills. A clue to the identity of these actors can be found in Bach (1983), where he notes that "most of the amendments in the nature of substitutes [i.e., substitute bills] offered in the Senate are committee amendments" (Bach, 1983, p. 586). Indeed, in the example cited at the beginning of the paper, the Committee on Veterans' Affairs reported both a bill and a substitute bill to the floor of the Senate. This then provides further evidence of the relative power of committees in their substantive domain.

One of the restrictive assumptions in the above model of voting procedures is that the set of alternatives is fixed prior to any decisions on agendas. Thus, future work will focus on the endogenous determination of alternatives, given that the two-stage amendment procedure will be employed, in much the same way Shepsle and Weingast (1984), Austen-Smith (1986), and Banks and Gasmi (1987) analyze endogenous agenda formation in the one-stage amendment procedure. The results in the current paper will obviously form the backbone of any such analysis, for to determine which alternatives should be placed on the agenda one must be able to solve for the eventual outcome for any such alternative. Another restriction to be relaxed in future work was that substitute amendments were not themselves the target of amendments; that is, decisions subsequent to 
the adoption of Rule 19 in the House allow there to be a bill, an amendment, an amended amendment, a substitute amendment, and an amended substitute amendment, along with the same possibilities related to a substitute bill. Specification of such a procedure would involve again a partition of the alternatives and orders on each element of the partition, but would also include a description of the order in which the alternatives resulting from the votes on the subsets are voted. For example, if there are three subsets of alternatives, two referring to the original bill and the remaining subset referring to a substitute bill, then the resulting alternatives from the first two subsets should be paired off prior to consideration of the third, while if two subsets refer to the substitute, the resulting alternative from the perfecting of the original bill (i.e., from the first subset) would wait until the winners from the remaining subsets are paired off (Sullivan, 1984; Bach, 1983). This added parameter in the procedure will doubtless complicate extensions of the current analysis; however, its relevance to the "real world" of congressional decision making would make such a task worthwhile.

\section{Manuscript submitted 9 February 1987}

Final manuscript received 15 October 1987

\section{REFERENCES}

Austen-Smith, David. 1987. Endogenous agenda formation under incomplete information. Mimeo., University of Rochester.

Bach, Stanley. 1983. Parliamentary strategy and the amendment process: Rules and case studies of congressional action. Polity, 15:573-92.

Banks, Jeffrey S. 1985. Sophisticated voting outcomes and agenda control. Social Choice and Welfare, 1:295-306.

Banks, Jeffrey S., and Farid Gasmi. 1987. Endogenous agenda formation in three-person committees. Social Choice and Welfare, 4:133-52.

McGarvey, David. 1953. A theorem on the construction of voting paradoxes. Econometrica, $21: 608-10$.

McKelvey, Richard. 1986. Covering, dominance, and institution-free properties of social choice. American Journal of Political Science. 30:283-314.

McKelvey, Richard, and Richard Niemi. 1978. A multistage game representation of sophisticated voting for binary procedures. Journal of Economic Theory, 18:1-22.

Miller, Nicholas. 1980. A new solution set for tournaments and majority voting: Further graphtheoretic approaches to the theory of voting. American Journal of Political Science, 24:68-96.

Ordeshook, Peter, and Thomas Schwartz. 1987. Agendas and the control of political outcomes. American Political Science Review, 81:179-200.

Shepsle, Kenneth, and Barry Weingast. 1984. Uncovered sets and sophisticated voting outcomes with implications for agenda institutions. American Journal of Political Science, 28:49-74.

Sullivan, Terry. 1984. Procedural structure: Success and influence in Congress. New York: Praeger. 